\title{
Putting a spin on photons
}

\author{
S. Burk, H. Fedder, J. Wrachtrup
}

S. Burk, H. Fedder, J. Wrachtrup, "Putting a spin on photons," Proc. SPIE 10329, Optical Measurement Systems for Industrial Inspection X, 1032906 (26 June 2017); doi: 10.1117/12.2278897

SPIE. Event: SPIE Optical Metrology, 2017, Munich, Germany 


\title{
Putting a spin on photons
}

\author{
S. Burk, H. Fedder, J. Wrachtrup ${ }^{a}$ \\ ard Institute of Physics and Institute for Quantum Science and Technology, IQST, University of \\ Stuttgart, Germany;
}

\begin{abstract}
We demonstrate a new tool to measure magnetic properties with photons. By using defects in diamonds we measure quantum properties so various quantities and demonstrate quantum precision sensing.
\end{abstract}

Keywords: Photochromism, single quantum system

\section{INTRODUCTION}

The nitrogen vacancy (NV) center in diamond is a promising candidate for emerging quantum technologies, such as quantum information processing, quantum communication and quantum sensing. Over the past years a series of key elements of scalable quantum information networks based on the NV center have been demonstrated, including single shot optical spin readout [1][2], a coherent spin-photon interface [3][4] and long lived stationary quantum registers. The latter are typically implemented using proximal nuclear spins provided by the diamond crystal lattice [5]. In this spin cluster architecture, the NV center's electron spin acts both as a central spin for optical readout and as a mediator for two qubit gates between nuclear spins. At the same time, the electron spin causes decoherence of the nuclear spins. Specifically, the electron spin limits the nuclear spin coherence time T2 to the electron spin T1 time [6]. This can be improved by averaging out the coupling between the electron and nuclear spin on a time scale faster than the hyperfine interaction, achieving a motional narrowing effect [6]. However, in this case, the coherence time is still limited by the NV center's electron spin.

With phosphorous donors in silicon, it was shown that the electron spins can be removed by ionizing the donor, and thereby nuclear spin memory times close to $1 \mathrm{~h}$ can be achieved at room temperature [7]. Here we demonstrate controlled and reversible switching of a single NV center from the NV- charge state to an ionized charge state that we attribute to $\mathrm{NV}+$. We show that the new charge state has no net electron spin, which eliminates electron spin induced decoherence of the nuclear spin. To switch the charge state on time scales comparable to the nuclear spin Larmor precession, we exploit P-I-P type double junction surface diamond diodes. Our results pave the way towards ultra-long lived room temperature quantum memories.

\section{EXPERIMENTS}

For our experiments we fabricate planar P-I-P type double junction diodes by spatially selective hydrogen and oxygen surface plasma treatment of a diamond sample using micro lithography. The hydrogen surface termination results in a two-dimensional hole gas below the diamond surface that provides p-type conduction, whereas the oxygen terminated surface provides an intrinsic region.

Details of the fabrication method and typical device characteristics are described elsewhere [8]. In this paper we exploit a [111] cut diamond to improve the optical collection efficiency from single NV centers and to facilitate the alignment of the magnetic field. Shallow NV centers (around $30 \mathrm{~nm}$ below the surface) were created prior to the fabrication of the P-I$\mathrm{P}$ structures by $6 \mathrm{keV} \mathrm{He}+$ ion implantation and subsequent annealing at $850 \mathrm{C}$. Figure $1 \mathrm{a}$ ) shows a schematic of the device. Hydrogen terminated areas are p-type conducting. The finger structure corresponds to a planar P-I-P type diode with a $1 \mu \mathrm{m}$ wide intrinsic channel. We apply bias voltages in the range -10 to $10 \mathrm{~V}$ across the intrinsic channel. Figure 1 b) shows the corresponding I-V characteristics. At voltages $>1 \mathrm{~V}$ the double junction diode shows the typical quadratic and linear regimes corresponding to the space charge limited current regime and velocity saturation regime, respectively, described by the Mott-Gurney law [9]. Figure $1 \mathrm{c}$ ) shows a confocal fluorescence microscope image zoomed in on the finger structure (excitation: $520 \mathrm{~nm}$ diode laser, detection: $650 \mathrm{~nm}$ long pass filter). Single NV centers are clearly visible. The purple transparent overlay indicates the hydrogen terminated surface areas of the finger structure. We first select NV

Optical Measurement Systems for Industrial Inspection X, edited by Peter Lehmann, Wolfgang Osten, Armando Albertazzi Gonçalves Jr., Proc. of SPIE Vol. 10329, 1032906 · @ 2017 SPIE CCC code: $0277-786 \mathrm{X} / 17 / \$ 18 \cdot$ doi: $10.1117 / 12.2278897$ 
centers that are suitable for charge state switching and electron spin resonance, by identifying centers that a) are mainly in the NV- state at zero bias voltage b) are mainly optically dark at a large applied bias voltage c) are oriented perpendicular to the sample surface and d) show a strong ODMR contrast at zero applied bias voltage.

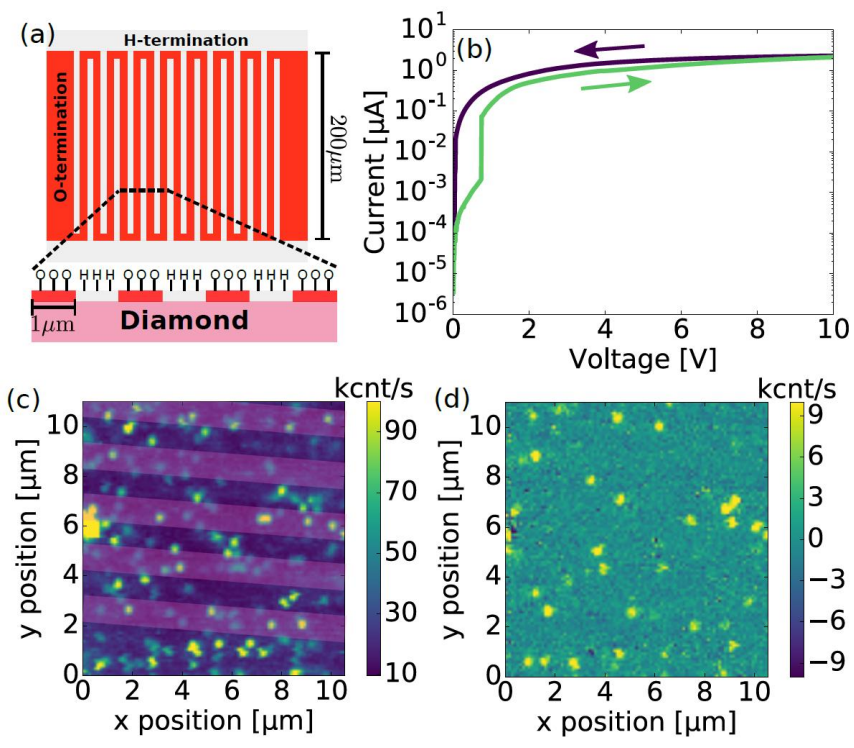

Figure 1. a) Schematic of the planar P-I-P double junction diamond diode. b) I-V characteristics. c) confocal image without applied bias voltage, the transparent purple overlay indicates the hydrogen terminated areas. d) fluorescence differences image acquired with bias voltages $+7.75 \mathrm{~V}$ and $-7.75 \mathrm{~V}$.

To achieve this, we acquire a microscope image of the fluorescence intensity difference between positive $(+7.75 \mathrm{~V})$ and negative $(-7.75 \mathrm{~V})$ bias voltage (Figure $1 \mathrm{~d})$ ). To avoid artifacts due to sample drift, the image was recorded by periodically switching the bias voltage on a time scale much smaller than the pixel dwell time and capturing the data accordingly. NV centers with a large fluorescence contrast appear predominantly at the edges between p-type and intrinsic regions. Subsequently we probe the ODMR contrast and fluorescence emission spectra of candidate NV centers. In this way we are typically able to identify NV centers that have an ODMR contrast in excess of $20 \%$ and optical spectra characteristic for NV- at zero applied bias voltage and a fluorescence intensity drop by at least $50 \%$ at higher bias voltage. Next, we measure NMR spectra by single shot nuclear spin readout [10] and vary the bias voltage. Figure 2 c) illustrates the measurement sequence. We begin with the NV center in the negative charge state (zero applied bias voltage) and perform single shot nuclear spin readout of the $14 \mathrm{~N}$ Nitrogen nuclear spin state by repeated application of a selective $\pi$-pulse on the $\mathrm{ms}=0 ; \mathrm{mI}=0$ to $\mathrm{ms}=-1 ; \mathrm{mI}=0$ electron spin transition followed by optical readout of the electron spin [1]. This projects the $14 \mathrm{~N}$ nuclear spin into the measured state. Next, we apply a bias voltage and allow the system to relax its charge state. Subsequently, we apply an RF $\pi$-pulse of variable frequency and amplitude. Finally, we return to zero bias voltage and perform a second nuclear spin readout. By comparing the measured nuclear spin states of the first and second measurement, we obtain the nuclear spin flip probability. The nuclear spin flip probability unveils the charge state [10]. For a calibrated $\pi$-pulse, the spin flip probability on resonance is equal to the average population of the corresponding charge state. Due to the quadrupole splitting of the $14 \mathrm{~N}$ nuclear spin, each charge state has unique NMR transitions. This allows us to measure the average population of the three charge states quantitatively. Figure 2 a) shows NMR spectra measured with an applied magnetic field of 6546 Gauss at three distinct NMR peaks with increasing bias voltage. At zero bias voltage the typical mixture of NV- and NV0 is observed. At increasing bias voltage the NV0 peak becomes more dominant and a new NMR line appears at $6.634 \mathrm{MHz}$. The line corresponds to a quadrupole splitting of $-4.619 \mathrm{MHz}$. At the maximum bias voltage, $72 \% \pm 12 \%$ of the population is in the corresponding charge state. We attribute this quadrupole splitting with the ionized NV center, $\mathrm{NV}+$. 

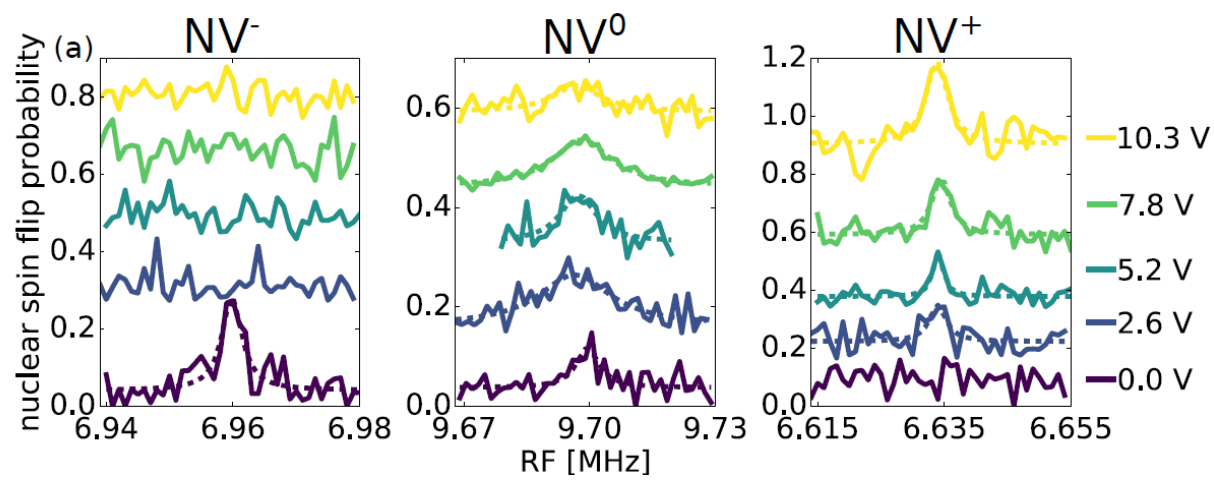

Figure 2. NMR spectra of $14 \mathrm{~N}$ with varying bias voltages. The NMR resonances correspond to: NV-, NV0 and NV+. Offsets have been added to the curves to allow stacking.

\section{CONCLUSION}

In conclusion, we have established controlled charge state switching of the NV-center in diamond to a new ionized state by means of hydrogen and oxygen surface terminated P-I-P type double junction diodes. We have identified the new charge state by single spin NMR measurements and attribute it to NV+. By quantifying the hyperfine enhancement of the nuclear spin Rabi frequency, we provide strong evidence that NV+ has no net electron spin. This agrees with our observed enhanced nuclear spin coherence time in the $\mathrm{NV}+$ charge state. Our results pave the way towards ultra-long lived room temperature quantum memories.

\section{REFERENCES}

[1] P. Neumann, J. Beck, M. Steiner, F. Rempp, H. Fedder, PR Hemmer, J. Wrachtrup, and F. Jelezko. Single-shot readout of a single nuclear spin. Science (New York, N.Y.), 329(5991):542-4, 2010.

[2] L. Robledo, L. Childress, H Bernien, B Hensen, P F Alkemade, and R Hanson. High-Fidelity projective readout of a solid-state spin quantum register. Nature, 477(7366):574-578, 2011.

[3] E Togan, Y Chu, a S Trifonov, L Jiang, J Maze, L Childress, MV G Dutt, a S Soerensen, P R Hemmer, a S Zibrov, and M D Lukin. Quantum entanglement between an optical photon and a solid-state spin qubit. Nature, 466(7307):730-734, 2010.

[4] H. Bernien, B. Hensen, W. Pfa_, G. Koolstra, M. S. Blok, L. Robledo, T. H. Taminiau, M. Markham, D. J. Twitchen, L. Childress, and R. Hanson. Heralded entanglement between solid-state qubits separated by three metres. Nature, 497(7447):86-90, 2013.

[5] G Waldherr, Y Wang, S Zaiser, M Jamali, T Schulte-Herbruggen, H Abe, T Ohshima, J Isoya, J F Du, P Neumann, and J Wrachtrup. Quantum error correction in a solid-state hybrid spin register. Nature, 506(7487):204-7,2014.

[6] P. C. Maurer, G. Kucsko, C. Latta, L. Jiang, N. Y. Yao, S. D. Bennett, F. Pastawski, D. Hunger, N. Chisholm,

M. Markham, D. J. Twitchen, J. I. Cirac, and M. D.Lukin. Room-Temperature Quantum Bit Memory Exceeding One Second. Science, 336(6086):1283-1286, 2012.

[7] K. Saeedi, S. Simmons, JZ. Salvail, P. Dluhy, H. Riemann, NV. Abrosimov, P. Becker, H-J. Pohl, JJL. Morton, and ML W. Thewalt. Room-temperature quantum bit storage exceeding 39 minutes using ionized donors in silicon-28. Science, 342(6160):830-3, 2013. [8] MV. Hauf, P. Simon, N. Aslam, M. Pfender, P. Neumann, S. Pezzagna, J. Meijer, J. Wrachtrup, M. Stutzmann, F. Reinhard, and JA. Garrido. Addressing single nitrogen-vacancy centers in diamond with transparent in-plane gate structures. Nano Letters, 14(5):23592364, 2014.

[9] A. A. Grinberg and Serge Luryi. Space-charge-limited current and capacitance in double-junction diodes. Journal of Applied Physics, 61(3):1181-1189, 1987.

[10] G. Waldherr, J. Beck, M. Steiner, P. Neumann, A. Gali, T. H. Frauenheim, F. Jelezko, and J. Wrachtrup. Dark states of single nitrogen-vacancy centers in diamond unravelled by single shot NMR. Physical Review Letters, 106(15):1-4, 2011. 